\title{
Propuesta de un marco de referencia de gestión de organizaciones usando Arquitectura Empresarial
}

\section{(Proposal of a referential Enterprise Architecture management framework for companies)}

\begin{abstract}
Cesar Esquetini Cáceres ${ }^{1}$, Oswaldo Moscoso Zea²
\section{Resumen:}

La Arquitectura Empresarial (AE) se concibe hoy en día, como una actividad esencial de gestión para visualizar y evaluar la dirección futura de una organización u compañía. Este documento tiene como objetivo realizar una revisión de literatura de AE para evaluar su rol como herramienta de gestión. Se detallará también como la $A E$ cumple dos propósitos fundamentales, primero como herramienta de diagnóstico institucional y autoevaluación para los procesos del negocio, aplicaciones e infraestructura tecnológica; segundo como herramienta para simular escenarios futuros que permita optimizar la toma de decisiones para la reestructuración y creación de planes de mejora. Además se analizan posibilidades para integrar AE con otras metodologías de gestión de negocios como el Cuadro de Mando Integral (Balanced Score Card) y el modelo europeo de calidad EFQM. Como resultado se presenta un marco de gestión de negocios con los elementos que se requieren para alcanzar estándares de excelencia y calidad en las organizaciones.
\end{abstract}

Palabras clave: Sistemas de Gestión, Arquitectura Empresarial, Cuadro de Mando Integral, EFQM.

\begin{abstract}
:
Enterprise Architecture (EA) is conceived nowadays as an essential management activity to visualize and evaluate the future direction of a company. The objective of this paper is to make a literature review on EA to evaluate its role as management tool. It is also explained how EA can fulfill two fundamental purposes, first as a tool for assessing the current situation (selfassessment) of an organization; second as a tool to model and simulate future scenarios that allow better decision making for the restructuration and development of improvement plans. Furthermore an analysis is made of the integration possibilities of EA with other business management methodologies, as balanced score card (BSC) and the model of the European Foundation for Quality Management (EFQM). As the result a management framework is presented, which includes the required elements to achieve excellence and quality standards in organizations.
\end{abstract}

Keywords: Management Systems; Enterprise Architecture; Balanced Score Card; EFQM.

\footnotetext{
${ }^{1}$ Universidad Tecnológica Equinoccial, Facultad de Ciencias Económicas y Negocios, Quito-Ecuador (cesquetini@ute.edu.ec; omoscoso@ute.edu.ec)

${ }^{2}$ Universidad Tecnológica Equinoccial, Facultad de Ciencias de la Ingeniería, Quito-Ecuador omoscoso@ute.edu.ec
} 


\section{Introducción}

En los últimos años la Arquitectura Empresarial $(A E)$ ha evolucionado de ser una disciplina netamente técnica que visualiza el desempeño de la infraestructura tecnológica de una empresa a convertirse en una disciplina gerencial que permite visualizar el estado actual de una organización a través de modelos (actuales) y evaluar la dirección estratégica, planteando arquitecturas y modelos de escenarios (futuros) para una mejor toma de decisiones (Bricknal, Darrell, Nilsson, \& Pessi, 2011). La evolución de AE ha tenido un fuerte impulso al convertirse en una herramienta que podría resolver el problema de la alineación del negocio con las tecnologías de Información (TI). Al implementar AE la alineación deja de ser un concepto idealista y teórico, y se convierte en un concepto práctico y aplicable que permite a las organizaciones usar las tecnologías de información de acuerdo a sus prioridades estratégicas. Así lo muestra una encuesta realizada por (Infosys, 2009). La encuesta muestra que en las organizaciones que usan $\mathrm{AE}$ un $32 \%$ ha mejorado la satisfacción de sus clientes, un $28 \%$ ha reducido los costos de $\mathrm{Tl}$ y un $27 \%$ ha logrado la estandarización y mejora de procesos.

Para implementar AE se han desarrollado marcos de referencia (The Open Group, 2011a);(Zachman, 1987), lenguajes de modelamiento por ejemplo: archimate y EPC (Event-driven Process Chains) que abarcan objetos en todos los dominios de arquitectura (ver Figura 1) y que permiten armonizar y orquestar los procesos del negocio con las aplicaciones e infraestructura tecnológica. Los proyectos de implementación de AE pueden ser muy largos cuando se quiere abarcar todos los departamentos de una organización, es por esto que (Franken, van Dijk, \& van Gils, 2013) propone que la implementación de $A E$ use un enfoque ágil similar a aquellos del desarrollo de software. Además los autores indican que los ingredientes claves para esta implementación son: un marco de referencia, combinar procesos y modelamiento y priorizar áreas que demanden crear arquitecturas o trabajar incrementalmente en cada capa de dominio de arquitectura, añadiendo valor en cada paso.

En este estudio se analiza como estructurar un modelo de gestión con herramientas gerenciales para potenciar los beneficios de usar $A E$ y cubrir algunas de las deficiencias que otras herramientas lo realizan de mejor manera. Algunas de las áreas que no cubre $A E$ son las siguientes: no define un mecanismo de autoevaluación institucional, tampoco permite medir el desempeño operativo de la organización en el trayecto hacia la consecución de su visión institucional. Es por ello que se propone un marco de referencia que incluya la $A E$ en conjunto con el modelo EFQM para tener un mecanismo de autoevaluación y el Cuadro de Mando Integral o BSC para poder establecer indicadores y medir el desempeño operativo de la organización. Este marco de referencia se diseña con el objetivo central de otorgar a las organizaciones una guía para la gestión orientada a la calidad y excelencia. 


\subsection{Metas}

El objetivo de investigación es proporcionar un marco de referencia que permita una gestión organizacional de excelencia. La pregunta clave a responder con este trabajo es:

- ¿Cómo definir un marco de referencia que permita una gestión organizacional orientada a la excelencia y a la calidad?

Como objetivos específicos se revisa el rol de $\mathrm{AE}$ como herramienta de gestión, se analiza las herramientas o modelos de gestión existentes, se selecciona aquellos modelos que suplan las deficiencias de $\mathrm{AE}$ y que aporten información adicional para una gestión integral de organizaciones.

\subsection{Motivación}

Los avances científicos y desarrollos tecnológicos así como la globalización han dado lugar a un ambiente organizacional muy complejo (lacob, Jonkers, Quartel, Franken, \& van den Berg, 2012). Esto junto a las regulaciones impuestas por los gobiernos y los organismos de control hace que la implementación de cambios y planes de mejoras sea extremadamente difíciles de concretar debido a una falta de entendimiento de la organización como un todo (lacob et al., 2012).

La Arquitectura Empresarial puede ser vista como un mapa que incorpora métodos y técnicas, el mismo que permite visualizar de forma holística una organización por medio del uso de modelos, marcos de referencias (frameworks), principios y directrices. El entendimiento de este mapa permite controlar y disminuir la complejidad del entorno del negocio y la tecnología con el fin de mejorar el impacto de cambios en la organización. Al disminuir la complejidad se puede establecer la línea base institucional (diagnóstico actual) y proponer las mejores alternativas de solución para el escenario deseado. Estos beneficios no son suficientes para gestionar una organización de calidad es por ello que es necesario incluir metodologías adicionales que permitan desarrollar una autoevaluación y establecer mecanismos de medición y control a través indicadores para asegurar que la gestión institucional se lleve a cabo con visibilidad y excelencia.

\subsection{Estructura del Artículo}

La estructura de este artículo es la siguiente: en la Sección 2 se presenta la Arquitectura Empresarial como herramienta de gestión organizacional y se detalla como AE puede constituirse en un elemento esencial del marco propuesto en esta investigación. En la Sección 3 se detalla el la propuesta del marco de referencia que incluye el modelo EFQM y el Cuadro de Mando Integral, detallando sus componentes y mostrando sus similitudes y diferencias. Finalmente en la Sección 4 se presentan las conclusiones del trabajo. 


\section{Arquitectura Empresarial}

De acuerdo a Lankhorst (Lankhorst, 2009, p. 3) "Arquitectura Empresarial es un conjunto coherente de principios, métodos y modelos que son usados en el diseño y en la realización de la estructura organizacional empresarial, procesos de negocio, sistemas de información e infraestructura". Desde un punto de vista diferente de acuerdo a (Gartner Inc., 2013) AE tiene un rol estratégico en una organización, su definición es la siguiente "Arquitectura Empresarial es el proceso de traducir la visión del negocio y su estrategia en un cambio organizacional efectivo por medio de crear, comunicar y mejorar los requerimientos claves, principios y modelos que describen el estado futuro de la empresa y permiten su evolución"

Otra definición importante de AE está dada por la Oficina Federal de Gestión de Arquitectura Empresarial de los Estados Unidos (FEA Program Management Office, 2007, p. 2) "Arquitectura Empresarial es una práctica de gestión para maximizar la contribución de los recursos de una agencia, inversiones de infraestructura tecnológica y actividades de desarrollo de sistemas, para alcanzar sus metas de rendimiento (performance)"

Como se puede observar en las distintas definiciones, AE es una disciplina que abarca aspectos del negocio y técnicos, permite manejar la creciente complejidad y por ende se puede gestionar los cambios de una forma organizada, proporcionando una vista holística de la empresa que incluye sus componentes organizacionales y sus relaciones. Por lo tanto (de Vries \& van Rensburg, 2008, p. 2) dice que AE es vista como "una práctica de gestión que ayuda a mejorar el rendimiento de una organización". En la Figura 1 se muestra una pirámide con las capas de arquitectura de una organización como: personas, negocio, aplicaciones y tecnología. Las flechas circulares representan el proceso general para implementar $A E$ en una organización: involucrando a los stakeholders, establecer la estructura de gestión y control, definir el enfoque y la construcción de las arquitecturas actuales (línea base) y las arquitecturas futuras, desarrollo del plan de migración, uso y mantenimiento de AE.

\subsection{Importancia de $A E$}

La Arquitectura Empresarial juega un rol importante como herramienta conductora de un cambio organizacional efectivo. Hoy en día las organizaciones que no tienen un departamento de calidad, gestión por procesos o Arquitectura Empresarial se enfrentan al riesgo de desarrollar o comprar productos o servicios cuya funcionalidad esté duplicada, es incompatible o muy costosa de mantener y lo más importante que no está alineada a las prioridades estratégicas que dicta la planificación empresarial. La necesidad de $A E$ es progresivamente más alta dependiendo del tamaño, complejidad, y distribución geográfica de una organización (Niemann, 2005).

Una encuesta fue desarrollada por la empresa (Infosys, 2009) entre 173 Directores, Gerentes del negocio y de TI. La encuesta muestra los objetivos y los beneficios de AE en diferentes organizaciones, entre los más importantes se puede mencionar: mejor alineación del negocio con 
las $\mathrm{TI}$, estandarización y mejora continua de procesos, reducción de costos, y satisfacción del cliente (Ver Figura 2).

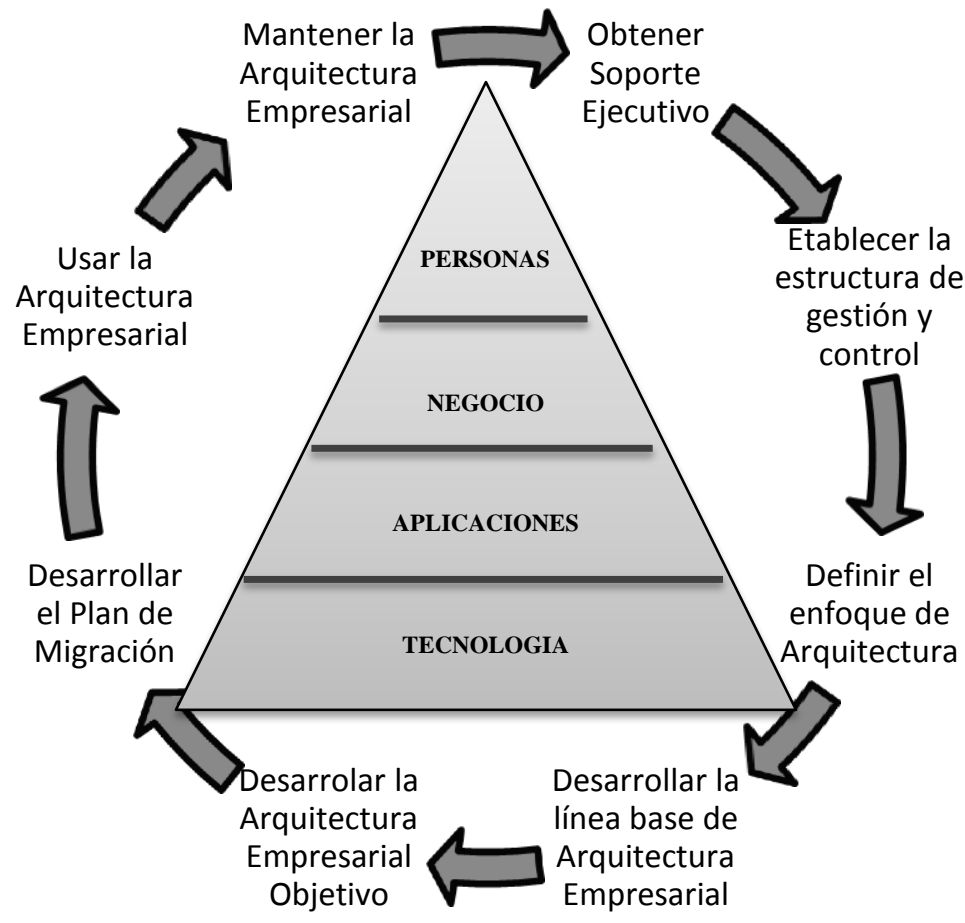

Figura 1. Arquitectura Empresarial Fuente: (Tucker \& Debrosse, 2003)

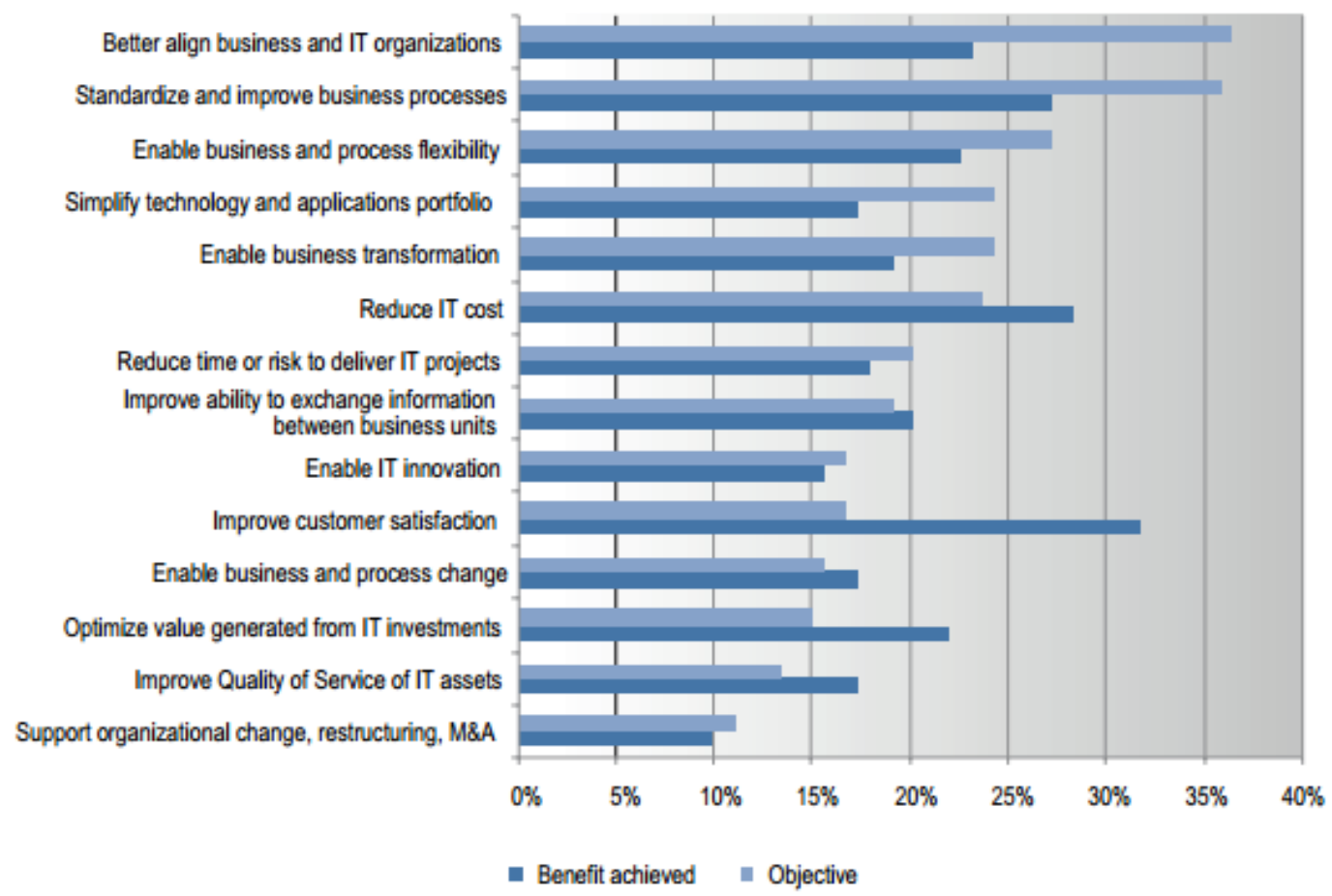

Figura 2. Objetivos y Beneficios de $A E$

Fuente: (Infosys, 2009) 


\subsection{Arquitectura Empresarial como herramienta de gestión}

Como se observa en los párrafos anteriores la importancia de AE va en aumento y los beneficios reales obtenidos a nivel del negocio son varios, por ejemplo: estandarizar y mejorar los procesos, permitir la flexibilidad empresarial, facilitar la transformación y restructuración del negocio, mejorar la satisfacción del cliente, mejorar la habilidad de intercambiar información entre las diferentes unidades del negocio. En síntesis luego de aplicar AE se puede observar sus resultados como herramienta de gestión, por un lado la visibilidad que otorgan los modelos de arquitectura permite evaluar el estado actual de la organización, por otro, la definición de escenarios futuros, permite tomar las mejores decisiones en proyectos de transición y cambio.

Para diseñar una $A E$ que sirva para la gestión del negocio, se requiere definir modelos para la línea base (situación actual), crear la hoja de ruta a seguir (análisis de brechas, planeación táctica y operativa) y diseñar modelos del escenario deseado de la empresa (la situación futura). Estas actividades deben contar con el apoyo de directivos de la empresa tanto del negocio como de $\mathrm{TI}$. El proceso de $A E$ tiene que ser comunicado a todos los niveles de la organización para poder capturar las necesidades y preocupaciones de los stakeholders. Además los esfuerzos arquitectónicos tienen que estar relacionados a la estrategia corporativa (Op't Land, Proper, Waage, Cloo, \& Steghuis, 2009). AE es esencial para organizaciones que quieren innovar implementando proyectos con sistemas de información para optimizar el valor del negocio (Roger, 2007). Más aún AE ayuda a entender y mejorar las interrelaciones entre los procesos del negocio, las aplicaciones, y la infraestructura técnica que las soporta (Schekkerman, 2011). Las aplicaciones claves de AE como herramienta de gestión se muestran en la Tabla 1.

Tabla 1. Aplicaciones claves de AE como herramienta de gestión

Fuente: (Op't Land et al., 2009)

\begin{tabular}{|c|c|}
\hline Aplicación & Descripción \\
\hline $\begin{array}{l}\text { Descripción de la Situación } \\
\text { Actual }\end{array}$ & Describir la situación actual y establecer un entendimiento en común. \\
\hline Dirección Estratégica & Describir diferentes escenarios de la situación futura de la organización. \\
\hline Análisis de Brecha & Identificación de desafíos y establecimiento de planes de migración \\
\hline Planeación Táctica & $\begin{array}{l}\text { Usar AE como herramienta de planeación para identificar la hoja de ruta } \\
\text { para las transformaciones. }\end{array}$ \\
\hline Planeación Operativa & $\begin{array}{l}\text { Usar AE como una herramienta de planeación para identificar proyectos } \\
\text { para la transformación. }\end{array}$ \\
\hline $\begin{array}{l}\text { Selección Parcial de } \\
\text { Soluciones }\end{array}$ & Usar AE para seleccionar soluciones, paquetes o servicios tercerizados. \\
\hline Arquitectura de Soluciones & Implementar soluciones por medio de gestión de proyectos. \\
\hline
\end{tabular}

Uno de los aspectos más importantes de AE es brindar soporte a los directivos y ejecutivos en la toma de decisiones con el objetivo de que la organización obtenga beneficios como: identificación de procesos duplicados y estandarización, ahorro en costos, además permite tener un 
entendimiento común de los recursos claves (Niemi, 2006). Por otra parte AE permite ir más allá de la gestión por procesos ya que involucra una serie de elementos adicionales para el análisis como: stakeholders, metas y estrategias, principios, aplicaciones e infraestructura. En este sentido la $A E$ permite dar respuesta a los retos más importantes que tienen un número alto de organizaciones como: fusiones, adquisiciones, apertura de nuevas sedes, introducción de nuevas tecnologías, presión para innovación, soluciones personalizadas y tercerización (Op’t Land et al., 2009).

Con el objetivo de gestionar las grandes cantidades de información para implementar AE de una manera eficiente, la organización debe usar un marco de trabajo (framework), una metodología y un software de AE (United States General Accounting Office, 2003). Un framework de arquitectura según el Open Group es "estructura base, o conjunto de estructuras que pueden ser usadas para el desarrollo de un gran número de diferentes arquitecturas..., debe describir un método para el diseño del estado futuro de la empresa en términos de componentes básicos, también debe contener un conjunto de herramientas, estándares y un vocabulario común" (The Open Group, 2011b). Para asegurar la calidad de la implementación de AE el uso de frameworks es indispensable. Los frameworks más conocidos para implementar AE son "The Open Group Architecture Framework" (TOGAF) y Zachman. Por el alcance del presente proyecto y por la necesidad de un método organizado y no de una taxonomía muy amplia como la representada por Zachmann (Microsoft, 2007) se escogerá TOGAF con su método de desarrollo de arquitecturas (ADM), el método tiene una serie de fases (Fase $A$ - fase $H$ ) como se muestra en la Figura 3 . Este método puede adaptarse de acuerdo a las necesidades y requerimientos del proyecto.

Sin embargo, pese a las grandes ventajas que ofrece esta herramienta uno de los problemas que se suscitan en su implementación operativa es la gran cantidad de información que se necesita procesar sobretodo en la definición, de las diferentes arquitecturas, de su estado actual y futuros, lo cual requiere de software especializado y capacidades de análisis de datos.

\section{Marco propuesto para la gestión de negocios}

Luego de analizar los beneficios y deficiencias de $A E$ y teniendo en mente que una gestión organizacional de excelencia tiene que ser medible, se introducen dos conceptos que permiten cubrir la necesidad de evaluar tanto el estado actual como el desempeño y logros para alcanzar la visión. Luego de una revisión de literatura que incluyó una investigación de las herramientas más usadas para este propósito se eligió el BSC y EFQM ya que permiten la autoevaluación y la medición del desempeño a través de indicadores, como eje fundamental de gestión. 


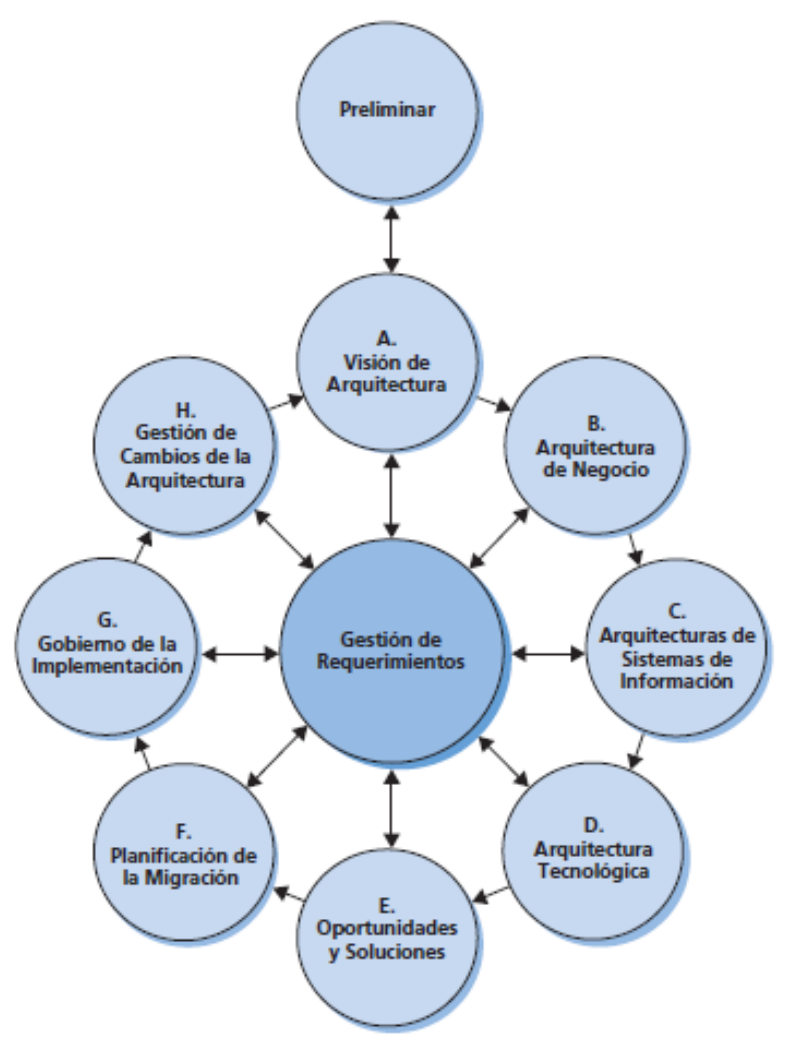

Figura 3. TOGAF ADM

Fuente: (Josey et al., 2013)

\subsection{Balanced Score Card (BSC)}

Esta herramienta fue presentada por (Kaplan \& Norton, 1992) como un instrumento que otorga elementos a los directivos de una empresa para medir el desempeño organizacional, sin tener que basarse únicamente en los criterios tradicionales de desempeño financiero como son el retorno de la inversión y las ganancias por acción. El balanced score card (BSC) conocido en español como "Cuadro de mando Integral" sugiere establecer indicadores para medir: satisfacción del cliente, procesos internos, mejora continua e indicadores financieros. De acuerdo a (Kaplan \& Norton, 1992) el BSC responde las siguientes preguntas: ¿Cómo nos ven nuestros clientes? (perspectiva del cliente), ¿En qué áreas tenemos que ser excelentes? (perspectiva interna), ¿Se puede continuar mejorando y creando valor? (perspectiva de innovación y aprendizaje), ¿Cómo nos ven los accionistas? (perspectiva financiera) Estas perspectivas se pueden observar en la Figura 4.

Desde el punto de vista de la perspectiva interna del BSC es importante dar prioridad a los procesos que tienen más impacto en la satisfacción de los clientes, entre los aspectos mencionados en el documento están: competencias y habilidades de los empleados, productividad y calidad (Kaplan \& Norton, 1992). Es indispensable también establecer medidas para las competencias importantes que debe alcanzar la organización. Las deficiencias del modelo es que los indicadores de gestión que se proponen deben ir de acuerdo a la priorización estratégica que 
es difícil identificar únicamente en el plan estratégico. La evaluación y autoevaluación que brinda AE y EFQM permite suplir esta deficiencia.

Los modelos que se obtengan con la AE más los objetivos de la planificación estratégica deben servir como las bases para identificar los procesos en los que se debe alcanzar excelencia y especificar medidas para alcanzar esos objetivos. Los directivos tienen que crear estas medidas y descomponer las mismas a los niveles operativos ya que los empleados en estos niveles pueden influenciar grandemente el cumplimiento de estas metas, posteriormente es importante analizar individualmente los indicadores para detectar aquellos que muestren señales de problemas para establecer correcciones y ver posibilidades de automatización.

\section{Balanced Score Card}

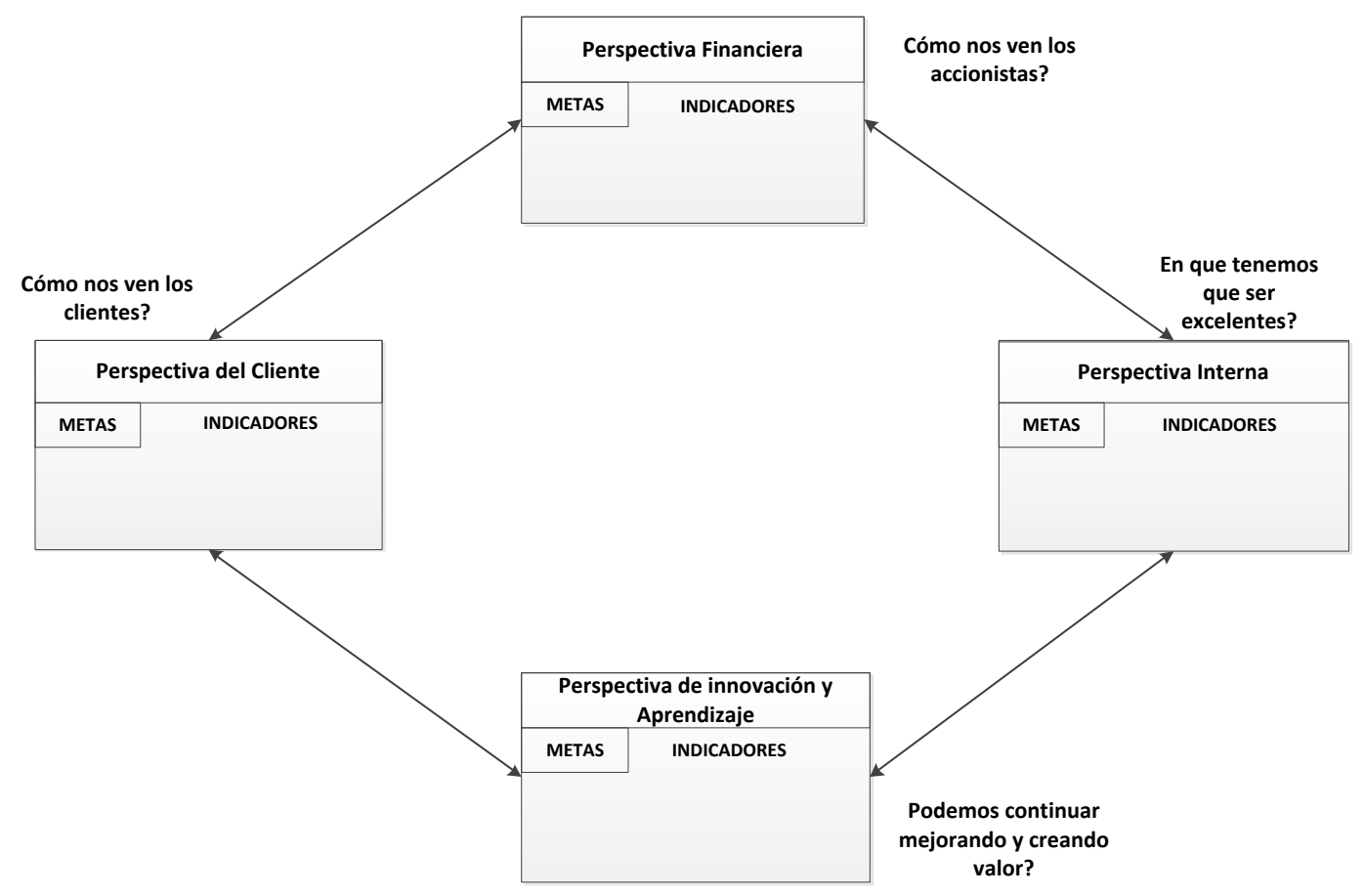

Figura 4. Balanced Score Card (Cuadro de Mando integral) Fuente: (Kaplan \& Norton, 1992)

\subsection{EFQM}

Es un modelo de calidad creado por la Fundación Europea para la Gestión de la Calidad (EFQM) organismo con sede en Bruselas, con alrededor de 500 socios y con presencia en más de 55 países(EFQM, 2012). Los principales criterios que toma en cuenta el EFQM se muestran en la Figura 5 y son: liderazgo, Política y Estrategia, Personas, Alianzas y Recursos, Procesos, Resultados en los Clientes, Resultados en las Personas, Resultados en la Sociedad y Resultados Clave 


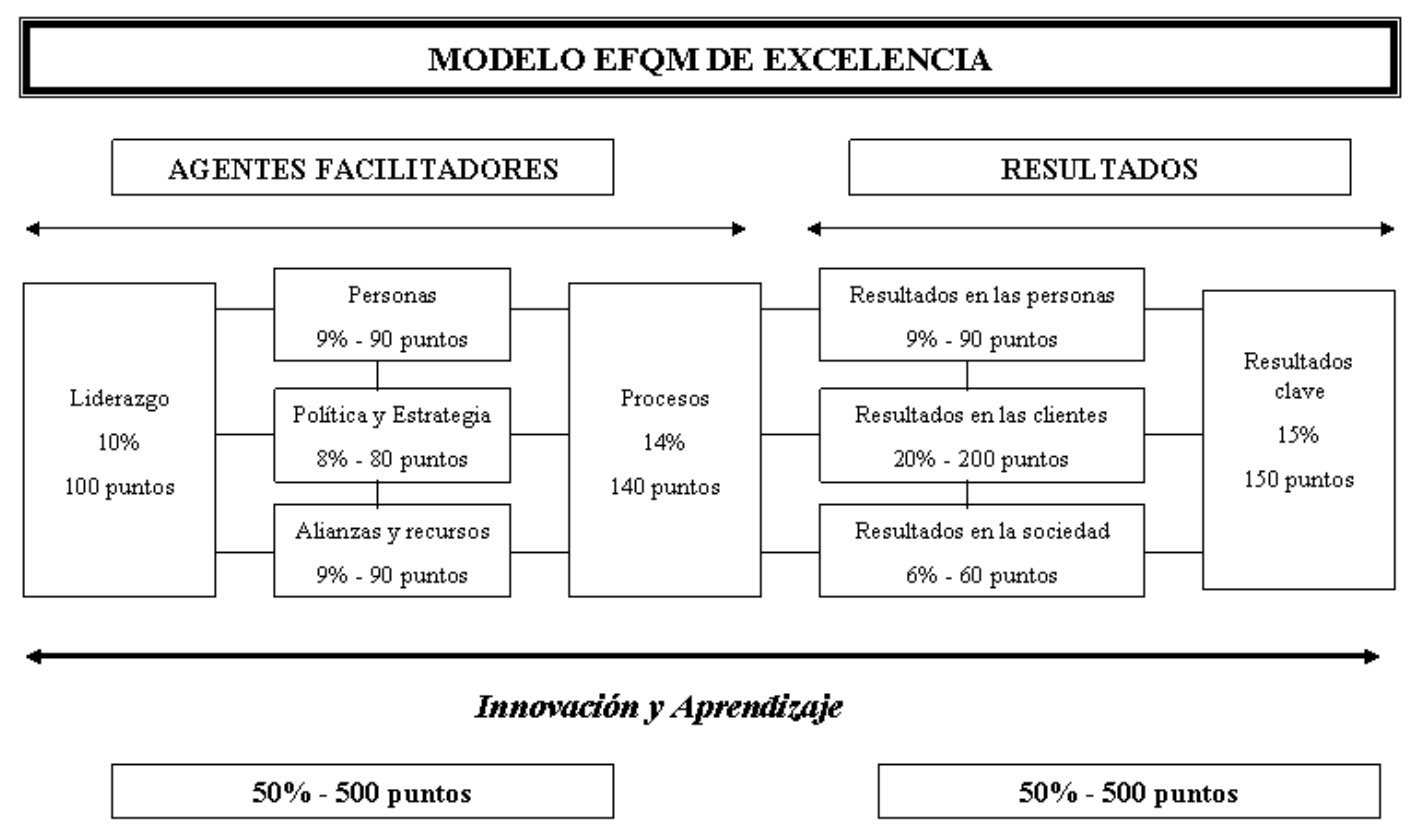

Figura 5. Modelo EFQM

Fuente: (EFQM, 2012)

EFQM tiene el objetivo de "evaluar el progreso de una organización hacia la excelencia" (Fundación Europea para la Gestión de la Calidad, 2003) e identificar las fortalezas de distintas perspectivas de la organización; en síntesis es un modelo que permite la autoevaluación organizacional. El modelo ve como el motor de la organización un liderazgo que transmita la visión, estrategia y políticas a través de las personas, las alianzas y recursos. Las perspectivas del modelo están agrupadas en dos categorías: Agentes Facilitadores y Resultados. Los Agentes Facilitadores permiten ver como la organización realiza actividades claves mientras la categoría Resultados permite ver cómo se van obteniendo dichos resultados.

EFQM tiene un componente importante denominado REDER cuyas siglas indican lo siguiente: Resultados, Enfoque, Despliegue, Evaluación y Revisión. Los cuatro últimos elementos son analizados en la categoría Agentes Facilitadores, mientras que el elemento Resultados se usa para evaluar la categoría Resultados.

Un tema importante a tomar en cuenta para el diseño del marco de referencia propuesto es la autoevaluación continua. Los beneficios que se pueden lograr como parte de la realización de una autoevaluación con EFQM son:

- Identificar áreas de fortaleza y de mejora y medir el progreso de las mismas de forma continua.

- Dar las pautas a la organización en la mejora de su modelo de gestión, relacionando los conceptos del modelo con las personas y sus responsabilidades.

- Difundir la excelencia como política de mejora continua en la organización. 
- Establecer comparaciones con organizaciones de la misma rama de acuerdo a la base de información de la Fundación sobre empresas que aplican el modelo.

EFQM identifica en qué nivel de madurez de excelencia se encuentra la organización y si la empresa desea ser evaluada puede obtener las siguientes distinciones en orden de importancia:

1. Premio Europeo a la Calidad

2. Reconocimiento a la Excelencia

3. Compromiso con la Excelencia

Como se mencionó el modelo toma en cuenta nueve criterios. Se considera que todos son de importancia para el desarrollo del modelo de gestión propuesto; sin embargo el modelo sugiere unos pesos y prioridades. Para el caso de este trabajo los criterios que adquieren una mayor importancia y por lo tanto requieren de un análisis más detallado son: Personas y Procesos (Fundación Europea para la Gestión de la Calidad, 2003).

\section{Criterio 3 Personas.}

Analiza la gestión del Recurso Humano de la empresa y los enfoques para la evaluación del desempeño, selección del personal, definición de puestos de trabajo, clima laboral, planes de incentivos, desarrollo profesional, entre otros. Es difícil un análisis de esta perspectiva sin la visibilidad de sus relaciones con otros dominios.

\section{Criterio 5 Procesos.}

Se basa en el análisis del modelo de gestión adoptado en la organización y como mejorar el mismo con el objetivo de satisfacer a los directivos. En esta perspectiva se evalúa si existe un diseño de procesos, si los procesos clave y los stakeholders han sido identificados, además es importante analizar la descripción de la necesidad que atiende el proceso así como verificar o definir indicadores. Adicionalmente en esta perspectiva se revisa si los procesos existentes tienen una orientación hacia los usuarios o beneficiarios de la institución.

Se recomienda abordar de manera especial estas perspectivas para el presente trabajo ya que son aquellas que están directamente relacionadas con los modelos de procesos resultados de la $\mathrm{AE}$, sin dejar de lado las otras perspectivas ya que de todas maneras en estudios posteriores será necesario realizar un análisis más detallado.

Para finalizar es importante resaltar que una de las características más interesantes del modelo EFQM es que permite combinarlo y potenciarlo con el marco de trabajo que sugiere la arquitectura empresarial sobre todo porque los dos (EFQM y AE) contemplan la gestión basado en procesos (Moreno Alego, 2007). La deficiencia que tiene el modelo EFQM está en la dificultad para evaluar sus criterios sin un conocimiento integral de la organización o sin una vista o modelo como lo otorga la $\mathrm{AE}$. 


\subsection{Análisis para la integración de herramientas}

Para establecer el mecanismo de hibridación para el marco propuesto, es importante el análisis en conjunto de los tres modelos explicados en las secciones anteriores como son la AE, BSC y el modelo EFQM.

Como se pudo observar en la Sección 0 el BSC realiza una revisión de cuatro perspectivas importantes de la organización: cliente, interna, innovación y financiera. El análisis de éstas, establece indicadores para medir el cumplimiento de las metas y los objetivos estratégicos. La perspectiva principal a tomar en cuenta para este trabajo es la perspectiva interna, la misma que identifica los procesos del negocio por medio de los cuales vamos a realizar los planes de mejora que influenciarán directamente las otras perspectivas, sobre todo la innovación en productos, servicios y modelos de negocio, y a su vez la optimización de los recursos financieros.

En cuanto al EFQM las perspectivas que presenta son: Liderazgo, Política y Estrategia, Personas, Alianzas y Recursos, Procesos, Resultados en los Clientes, Resultados en las Personas, Resultados en la Sociedad, Resultados Clave. La autoevaluación usando estas perspectivas puede apoyarse en AE para visualizar el funcionamiento actual de la organización.

Para lograr la combinación de estas tres herramientas, en primer lugar se toman como punto de partida los estudios realizados de integración entre el BS y el EFQM para luego incorporar a la AE. El análisis de (Lamotte \& Carter, 2000), muestra como el modelo EFQM y el BSC comparten algunas características en común, las más importantes que describe el autor son: se basan en indicadores, promueven un diálogo interno sobre mejoramiento del perfomance, funcionan como catalizadores entre el cambio y la acción. El éxito en la implementación depende en el compromiso de los directivos para la mejora continua. Un análisis que compara el BSC y EFQM se presenta a continuación (ver Tabla 2).

Como se observa en la tabla anterior EFQM y BSC comparten ciertos principios de gestión pero los modelos tienen diferentes orígenes, diferentes rutas para sus objetivos y otorgan diferentes beneficios y resultados. En esencia de acuerdo a (Lamotte \& Carter, 2000) la diferencia fundamental es que BSC fue diseñado para comunicar y evaluar el performance estratégico, mientras que EFQM junto con sus diversas aplicaciones incluidas la autoevaluación se enfoca en guiar en la adopción de mejores prácticas en todas las actividades de gestión de la organización. De acuerdo a (Martínez, 1999) el BSC y el EFQM son complementarios y permiten buscar el equilibrio entre la mejora, la calidad de los procesos y los resultados financieros de una organización. Ahora bien como características importantes de las dos herramientas se observa la siguiente comparación en la Tabla 3. 
Tabla 2. Comparando BSC y EFQM

Fuente: (Lamotte \& Carter, 2000)

\begin{tabular}{|c|c|c|}
\hline Criterio & BSC & EFQM \\
\hline Orígenes & Medir desempeño y creación de valor & Gestión de la Calidad Total \\
\hline $\begin{array}{l}\text { Aspiraciones y } \\
\text { Beneficios Buscados }\end{array}$ & $\begin{array}{l}\text { Mejorar el desempeño. } \\
\text { Traducir la estrategia organizacional en } \\
\text { indicadores operacionales, } \\
\text { cuantificables y enfocados. Impulsador } \\
\text { del desempeño estratégico. }\end{array}$ & $\begin{array}{l}\text { Mejorar el desempeño. } \\
\text { Identificar las fortalezas y áreas de } \\
\text { mejora a través de los procesos de la } \\
\text { organización para promover mejores } \\
\text { prácticas de gestión. } \\
\text { Impulsador de mejores prácticas de } \\
\text { gestión. }\end{array}$ \\
\hline Entregables & $\begin{array}{l}\text { Un conjunto de objetivos estratégicos } \\
\text { enlazados con indicadores / metas de } \\
\text { avance y atrasos en cuatro } \\
\text { perspectivas. } \\
\text { Un conjunto de iniciativas alineadas a } \\
\text { los objetivos estratégicos y métricas. }\end{array}$ & $\begin{array}{l}\text { Un punto de referencia para comparar } \\
\text { la evaluación de la calidad de una } \\
\text { organización, sus procesos y } \\
\text { resultados en } 9 \text { criterios del modelo. }\end{array}$ \\
\hline Enfoque de Desarrollo & $\begin{array}{l}\text { Impulsado por la estrategia, basado en } \\
\text { talleres, iterativo, impulsado por } \\
\text { hipótesis, participación del equipo } \\
\text { directivo, vistas macro y vistas a futuro. }\end{array}$ & 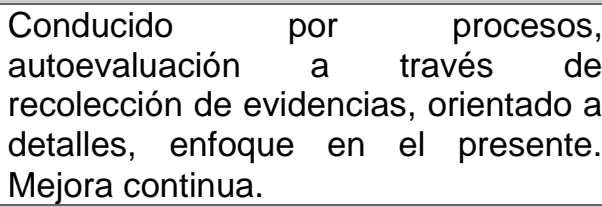 \\
\hline Factores de Éxito & $\begin{array}{l}\text { Compromiso y patrocinio del equipo } \\
\text { directivo. Proceso continuo inmerso en } \\
\text { los procesos de gobernanza. }\end{array}$ & $\begin{array}{l}\text { Compromiso y patrocinio del equipo } \\
\text { directivo. Proceso continuo inmerso } \\
\text { en la gestión del día a día. }\end{array}$ \\
\hline
\end{tabular}

Tabla 3. Comparación características importantes BSC, EFQM Fuente: (Lamotte \& Carter, 2000)

\begin{tabular}{|c|c|}
\hline $\begin{array}{l}\text { Basado en Aspiraciones - Vista a Futuro de } \\
\text { la compañía. } \\
\text { El BSC se crea con una visión de lo que quiere } \\
\text { lograr la compañía en un plazo de } 2-5 \text { años. }\end{array}$ & $\begin{array}{l}\text { Basado en lo Actual - Vista de la situación } \\
\text { actual de la compañía. } \\
\text { Los resultados de la autoevaluación describen } \\
\text { el estado actual de los procesos de la } \\
\text { organización. }\end{array}$ \\
\hline $\begin{array}{l}\text { Comienza analizando la visión y revisando el } \\
\text { presente para ver las brechas y el camino } \\
\text { estratégico a seguir. }\end{array}$ & $\begin{array}{l}\text { Identifica las fortalezas y las posibles áreas de } \\
\text { mejora dentro del conjunto de actividades } \\
\text { basadas en criterios objetivos. }\end{array}$ \\
\hline $\begin{array}{l}\text { Define los pasos para los cambios necesarios } \\
\text { de alto nivel (típicamente financieros) hacia } \\
\text { dónde quiere llegar la compañía a nivel } \\
\text { estratégico. }\end{array}$ & $\begin{array}{l}\text { Los resultados de la autoevaluación no juzgan } \\
\text { en que actividades se tiene que enfocar la } \\
\text { organización, basados en una vista a futuro. }\end{array}$ \\
\hline $\begin{array}{l}\text { No analiza la calidad de los procesos y } \\
\text { actividades actuales. }\end{array}$ & $\begin{array}{l}\text { Alienta la mejora continua en las operaciones } \\
\text { alineado a los conceptos fundamentales de } \\
\text { Gestión de la Calidad Total. }\end{array}$ \\
\hline
\end{tabular}

Del análisis anterior se observa que existe potencialidad para la hibridación de los tres modelos ya que para potenciar el uso de EFQM, BSC y de AE es importante visualizar la organización actual y futura, además la forma más adecuada para establecer mejoras en los procesos es mediante un mapa que muestre sus relaciones, sus entradas y sus salidas. Por una parte existen coincidencias entre las perspectivas del BSC, del EFQM, y actividades compatibles en la AE (para el análisis comparativo realizado ver Tabla 4) por otra parte la visibilidad para traducir la estrategia en mecanismos de medición y evaluación se puede obtener con el método de TOGAF ADM y con la definición de los mapas organizacionales y las arquitecturas. 


\subsection{Marco de Gestión Integrado (AE, BSC, EFQM)}

La Tabla 3 presenta pautas de cómo integrar la AE para la creación del modelo híbrido. Como se revisó la $A E$ permite obtener una vista a través de modelos de la situación actual de la compañía en diferentes dominios de negocio y tecnológicos, esto junto con la autoevaluación del EFQM permite identificar áreas de interés (fortalezas o debilidades). Por ende al juntar estas dos herramientas (EFQM y $\mathrm{AE}$ ) se tiene una visión mucho más amplia del manejo y desempeño actual de la organización. Esto a la vez permite una mejora en la toma de decisiones por parte de los directivos para poder construir un nuevo modelo de gestión organizacional o para asegurar que se alineen los objetivos estratégicos a los objetivos operacionales y estructura organizacional.

El análisis preliminar priorizar las actividades estratégicas en procesos y estructura organizacional y dará información para la correcta asignación de los recursos. Esta priorización enfoca a los procesos donde se tiene que realizar reingeniería y en donde existe el mayor potencial para poder introducir innovación y mejoras en la gestión institucional. La dirección estratégica es el valor agregado que tenemos al usar BSC junto con EFQM y AE.

Por otro lado, la AE visualiza el futuro de la organización también a través de modelos, con estos modelos a futuro se puede evaluar opciones, determinar la brecha existente y determinar el mejor camino para alcanzar el escenario deseado. En este aspecto el BSC permite traducir la visión que brinda la $A E$ en un cambio operacional efectivo a través de un enfoque estratégico claro y un plan de acción adecuado por medio de la implementación de indicadores de gestión. Más aún la Arquitectura Empresarial otorga información adicional para la priorización estratégica (árbol de prioridades) y también para la identificación de fortalezas y debilidades (modelos y vistas de arquitecturas de la situación actual). Además, otorga la visibilidad deseada de la organización a través de los modelos (modelos y vistas de arquitecturas de la situación futura - reingeniería de procesos). En la Tabla 4 se muestra un análisis comparativo de las interrelaciones de las tres herramientas y los mecanismos para compartir información en cada una de sus fases o perspectivas. Esta tabla se basa en un análisis previo para la gestión de universidades que incluye BSC y EFQM realizado por (Ticse \& Barreda, 2009).

De manera general el marco de referencia sugiere lo siguiente:

1. Realizar el levantamiento de la AE actual para determinar la línea base y establecer prioridades estratégicas.

2. Realizar una autoevaluación usando EFQM para determinar áreas de mejora y establecer prioridades estratégicas.

3. Realizar la propuesta de escenarios futuros mediante AE y establecer la hoja de ruta y el análisis de brecha.

4. Usar BSC para establecer indicadores en todas las perspectivas (BSC, EFQM) y poder medir la consecución de objetivos para alcanzar la visión institucional de la empresa. 
Tabla 4. Análisis Comparativo BSC, EFQM, AE

\begin{tabular}{|l|l|l|}
\hline Perspectiva del Cliente & Resultados en los Clientes & Fase B,C,D (Arquitecturas) \\
\hline Perspectiva Interna & Procesos & $\begin{array}{l}\text { Fase B (Arquitectura del } \\
\text { negocio) } \\
\text { Fase E (Oportunidades y } \\
\text { Soluciones) }\end{array}$ \\
\hline Perspectiva de Innovación & $\begin{array}{l}\text { Personas, Resultados en las } \\
\text { Personas }\end{array}$ & $\begin{array}{l}\text { Fase B (Arquitectura del } \\
\text { negocio) } \\
\text { Fase E (Oportunidades y } \\
\text { Soluciones) }\end{array}$ \\
\hline Perspectiva Financiera & Resultados Clave & $\begin{array}{l}\text { Preliminar, Fase A (Visión de la } \\
\text { Arquitectura) }\end{array}$ \\
\hline & Liderazgo & \\
\hline & Política y Estrategia & \\
\hline & Alianzas y Recursos & \\
\hline
\end{tabular}

El marco de referencia es ilustrado en la Figura 6. Propone una gestión integral que busca aprovechar las potencialidades de cada modelo y crear sinergia para poder alcanzar estándares de calidad y excelencia. El proceso es iterativo en el tiempo y toma como entradas los requerimientos de los stakeholders y la planificación estratégica organizacional.

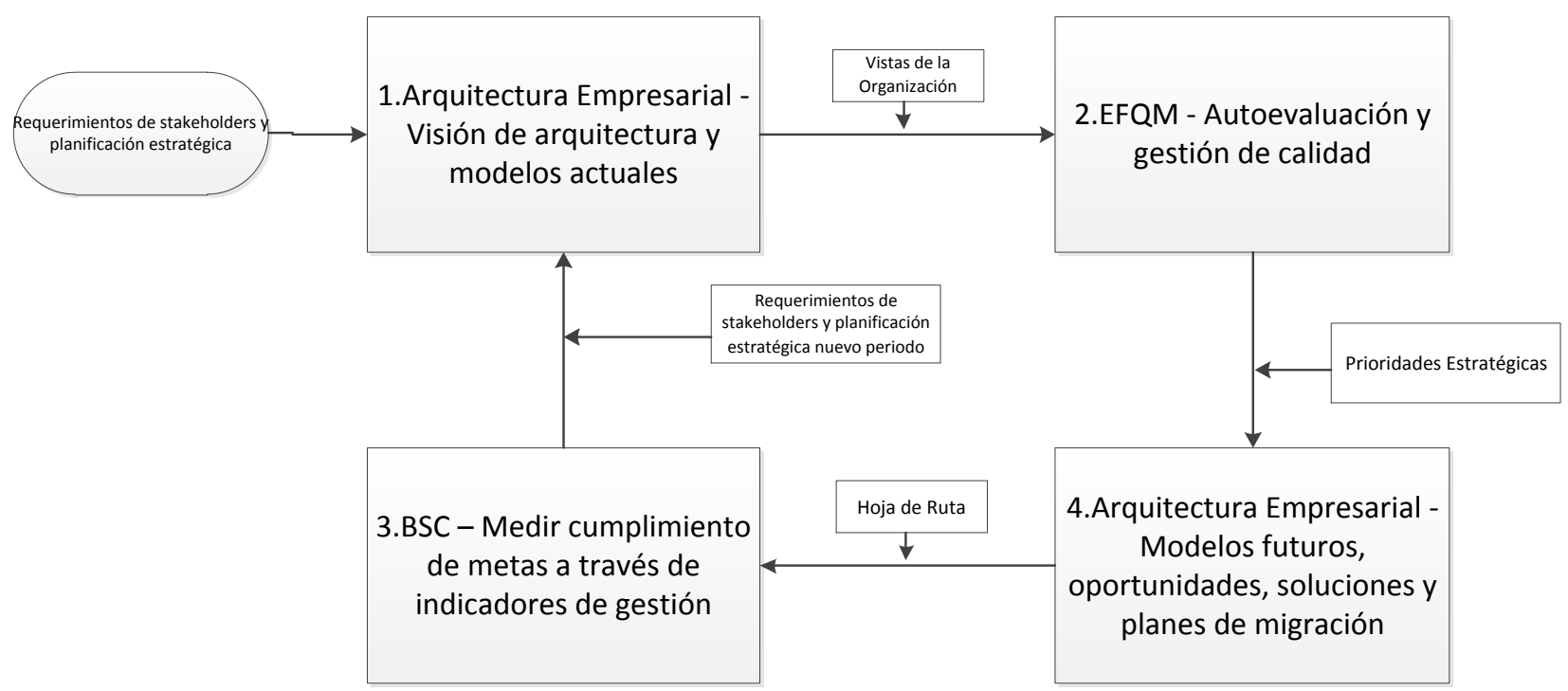

Figura 6. Marco de Gestión Integrado (AE,BSC,EFQM)

A continuación se describe el proceso detallado del marco de referencia:

1. Con los requerimientos claros se debe crear la $A E$ usando el framework seleccionado ADM. El ADM propone en la Fase Preliminar determinar las Capacidades Arquitectónicas deseadas por la organización además en su fase A (Visión de la Arquitectura) se desarrolla una visión de alto nivel de las capacidades que se desea obtener con la $A E$. Posteriormente se implementa la Fase B, C, D es decir la creación de los modelos de arquitectura. Estos modelos abarcan el dominio del negocio, sistemas de información y 
arquitectura tecnológica, en este primer paso se obtiene como entregables la línea base y vistas de la organización.

2. En el paso dos los entregables sirven como entradas para la autoevaluación que se realiza usando el modelo de calidad y excelencia EFQM, está autoevaluación sugiere ponderar las nueve perspectivas que ofrece el modelo. Para esto es importante definir un equipo de evaluación experto y entregarles como insumos los modelos y vistas de la organización obtenidos en el paso uno, además, compartir toda la información operativa, financiera y estratégica necesaria para que la evaluación arroje resultados óptimos. Como entregables de esta fase se obtiene la autoevaluación institucional la misma que junto con los modelos permiten ser insumos de análisis para identificar prioridades estratégicas.

3. Una vez identificado las prioridades estratégicas de la organización se debe trabajar en los planes de mejora e identificar los mecanismos de migración adecuados. Aquí se define nuevamente arquitecturas usando ADM como método guía tanto para las fases B, C y D pero en este paso las vistas que se obtendrán de los modelos permitirán ver escenarios futuros. Con los modelos actuales y futuros se determinará la hoja de ruta para poder efectuar cambios. Posteriormente, se implementa la fase $E$ del ADM (Oportunidades y Soluciones), en esta fase se realiza análisis de brechas y por ende se identifica los proyectos o programas a implementar. También se ejecuta la fase $\mathrm{F}$ (Planificación de la Migración) donde se establece el plan a seguir para la implementación de los cambios. Finalmente es necesario implementar la fase $G$ (Gobierno de Implementación) y la fase H (Gestión de la Arquitectura) para poder definir estructuras de gobierno y asegurar que los cambios se gestionen de una manera controlada.

4. Una vez definidos explícitamente cuáles son las metas de la organización y cuáles son los proyectos a implementar (hoja de ruta), es importante medir el cumplimiento de las mismas. Para ello se utiliza el BSC y se definen indicadores de gestión para revisar que lo propuesto se cumpla de forma efectiva. Estos indicadores usan las perspectivas propias del BSC pero además se sugiere incorporarlos en las perspectivas adicionales presentadas en el modelo EFQM.

\section{Conclusiones}

En este trabajo se ha presentado una propuesta metodológica de gestión que permite establecer: el diagnóstico institucional, la autoevaluación, la medición de desempeño, la situación futura y la correcta planeación de las mejoras a realizarse y su correspondiente seguimiento.

La parte central de este trabajo corresponde al marco de referencia para la gestión organizacional orientada a la calidad y la excelencia y su aplicación. La validación fue realizada en una institución privada de educación superior. 
La suma sinérgica conseguida con la inclusión de AE, EFQM y BSC permite potencias las fortalezas de cada una de las herramientas incorporadas, mitigar debilidades individuales y cubrir más dimensiones en el análisis de la empresa, de acuerdo a lo mostrado en la investigación.

La arquitectura de los procesos del negocio posibilita, a través de los modelos de AE tener visibilidad para incorporar un sinnúmero de mejoras: reducción de costos innecesarios (estandarización y restructuración), posibilidad de establecer indicadores por proceso, mejoras o cambios en la estructura organizacional. Por otra parte es importante aprovechar los modelos de "AE a futuro" ya que estos establecen los posibles escenarios deseados y analizan cuáles son los más convenientes y estratégicamente los más importantes de implementar.

A pesar de las ventajas que brinda la $A E$, tiene algunas deficiencias que son necesarias considerar. Entre las más importantes para la gestión organizacional están: no define un mecanismo de autoevaluación institucional, tampoco permite medir el desempeño operativo de la organización, además el procesamiento de una arquitectura global requiere mucho esfuerzo para procesamiento de información y recurso humano altamente capacitado en los dominios de arquitectura. Es por ello que al complementar AE con BSC y EFQM se logra potenciar la gestión y suplir ciertas deficiencias

El alinear la estrategia del negocio con los procesos y con TI permite realizar una reingeniería de procesos basada en áreas estratégicas que requieran automatización y que aporten a la visión del negocio, la autoevaluación con EFQM ayuda a identificar estas áreas de priorización. La estrategia debe definir los procesos claves del negocio es por esto que es necesario tener presente siempre la relación existente entre los distintos dominios de arquitectura con la estrategia y establecer indicadores usando BSC para poder asegurar el cumplimiento de objetivos y la correcta alineación de los objetivos estratégicos con los objetivos operativos y tácticos.

Se concluye que existe un gran potencial en el uso de este marco de gestión propuesto por lo cual se recomienda su uso y adaptación de acuerdo a las necesidades específicas de cada organización.

\section{Bibliografía}

Bricknal, R., Darrell, G., Nilsson, H., \& Pessi, K. (2011). Enterprise Architecture: Critical Factors affecting modelling and management (p. 2). Retrieved from http://pdf.aminer.org/000/245/735/enterprise_architecting_critical_problems.pdf

EFQM. (2012). Modelo EFQM. Retrieved August 15, 2014, from http://www.efqm.es/

FEA Program Management Office. (2007). FEA Practice Guidance, (November). 
Franken, H., van Dijk, S., \& van Gils, B. (2013). Three Best Practices for Successful Implementation of Enterprise Architecture Using the TOGAF® Framework and the ArchiMate ${ }^{\circledR}$ Modeling Language. The Open Group Blog. Retrieved from http://blog.opengroup.org/2013/02/08/three-best-practices-for-successful-implementation-ofenterprise-architecture-using-the-togaf-framework-and-the-archimate-modeling-language/

Fundación Europea para la Gestión de la Calidad. (2003). EFQM Introducción a la Excelencia. Bruselas Bélgica.

Gartner Inc. (2013). Gartner IT Glossary. Retrieved March 18, 2013, from http://www.gartner.com/it-glossary/enterprise-architecture-ea/

lacob, M.-E., Jonkers, H., Quartel, D., Franken, H., \& van den Berg, H. (2012). Delivering Enterprise Architecture with TOGAF and Archimate.

Infosys. (2009). Enterprise Architecture Expands its Role in Strategic Business Transformation Survey Report. Architecture.

Josey, A., Harrison, R., Homan, P., Rouse, M., van Sante, T., Turner, M., \& van der Merwe, P. (2013). Togaf guía de bolsillo. NL: Van Haren.

Kaplan, R. S., \& Norton, D. P. (1992). The Balanced Scorecard - Measures that Drive Performance. Harvard Business Review Feb - 1992.

Lamotte, G., \& Carter, G. (2000). Are the Balanced Scorecard and the EFQM Excellence Model mutually exclusive or do they work together to bring added value to a company? EFQM Common Interest Days. Retrieved from http://www.paceperformance.co.im/documents/Link b EFQM and Balanced Scorecard V5.pdf

Lankhorst, M. (2009). Enterprise Architecture at Work Modelling Communication and Analysis (2nd ed.). Springer Dordrecht.

Martínez, R. (1999). EL BALANCED SCORECARD (BSC) Y SUS RELACIONES CON LA CALIDAD. Seminario Internacional "EI BSC y su relación con la gerencia estratégica y los indicadores de gestión", 1-7.

Microsoft. (2007). A Comparison of the Top Four Enterprise-Architecture Methodologies. Retrieved from http://msdn.microsoft.com/en-us/library/bb466232.aspx

Moreno Alego, J. (2007). Guía para la aplicación del modelo EFQM de Excelencia en Entidades de Acción Social. Cuaderno de Gestión 2, Fundación Luis Vives. 
Niemann, K. D. (2005). From Enterprise Architecture to IT Governance.

Niemi, E. (2006). Enterprise Architecture Benefits : Perceptions from Literature and Practice. 7th IBIMA Conference Internet \& Information Systems in the Digital Age (pp. 14-16). Brescia, Italy.

Op't Land, M., Proper, E., Waage, M., Cloo, J., \& Steghuis, C. (2009). Enterprise Architecture Creating Value by Informed Governance. Springer-Verlag.

Roger, S. (2007). A Comparison of the Top Four Enterprise Architecture Methodologies. MSDN Library. Retrieved April 16, 2013, from http://msdn.microsoft.com/en-us/library/bb466232.aspx

Schekkerman, J. (2011). Enterprise Architecture Tool Selection Guide. Institute for Enterprise Architecture Developments.

The Open Group. (2011a). TOGAF ® Version 9.1. Retrieved April 12, 2013, from https://www2.opengroup.org/ogsys/jsp/publications/PublicationDetails.jsp?catalogno=I

The Open Group. (2011b). TOGAF ® Version 9.1. Retrieved April 12, 2013, from https://www2.opengroup.org/ogsys/jsp/publications/PublicationDetails.jsp?catalogno=I

Ticse, E., \& Barreda, H. (2009). METODO INTEGRADO PARA LA GESTION DE UNIVERSIDADES BASADO EN EL BALANCED SCORECARD (BSC) Y EL MODELO EUROPEO DE CALIDAD (EFQM): CASO U.C.S.M. IX Coloquio Internacional sobre Gestión Universitaria en América del Sur.

Tucker, R., \& Debrosse, D. (2003). Enterprise Architecture Roadmap for Modernization. Mitre Corporation. Retrieved from http://www.mitre.org/news/the_edge/fall_03/tucker.html

United States General Accounting Office. (2003). A Framework for Assessing and Improving Enterprise Architecture Management, (April). Retrieved from http://www.gao.gov/new.items/d03584g.pdf

De Vries, M., \& van Rensburg, A. (2008). Enterprise Architecture - New business value perspectives. Southafrican Journal of Industrial Engeneering, 19, 2. Retrieved from http://sajie.journals.ac.za/pub/article/view/103/99

Zachman, J. a. (1987). A framework for information systems architecture. IBM Systems Journal, 26(3), 276-292. Retrieved from http://ieeexplore.ieee.org/lpdocs/epic03/wrapper.htm?arnumber=5387671 